\title{
Confronting Surface Hopping Molecular Dynamics with Marcus Theory for a molecular donor-acceptor system
}

\author{
Jacob Spencer ${ }^{1}$, Laura Scalfi, ${ }^{1,2}$, Antoine Carof $^{1}$, Jochen Blumberger ${ }^{1 *}$ \\ June 20, 2016 \\ ${ }^{1}$ Department of Physics and Astronomy, University College London, Gower Street, London WC1E 6BT, UK. \\ ${ }^{2}$ Department of Chemistry, École Normale Supérieure - PSL Research University, 24 rue Lhomond, 75005 Paris, France \\ *e-mail: j.blumberger@ucl.ac.uk
}

\begin{abstract}
We investigate the performance of fewest switches surface hopping (SH) in describing electron transfer (ET) for a molecular donor-acceptor system. Computer simulations are carried out for a wide range of reorganisation energy $(\lambda)$, electronic coupling strength $\left(H_{\mathrm{ab}}\right)$ and driving force using our recently developed fragment orbital-based $\mathrm{SH}$ approach augmented with a simple decoherence correction. This methodology allows us to compute SH ET rates over more than four orders of magnitude, from the sub-picosecond to the nanosecond time regime. We find good agreement with semi-classical ET theory in the non-adiabatic ET regime. The correct scaling of the SH ET rate with electronic coupling strength is obtained and the Marcus inverted regime is reproduced, in line with previously reported results for a spin-boson model. Yet, we find that the SH ET rate falls below the semi-classical ET rate in the adiabatic regime, where the free energy barrier is in the order of $k_{\mathrm{B}} T$ in our simulations. We explain this by first signatures of non-exponential population decay of the initial charge state. For even larger electronic couplings $\left(H_{\mathrm{ab}}=\lambda / 2\right)$, the free energy barrier vanishes and ET rates are no longer defined. At this point we observe a crossover from ET on the vibronic time scale to charge relaxation on the femtosecond time scale that is well described by thermally averaged Rabi oscillations. The extension of the analysis from the non-adiabatic limit to large electronic couplings and small or even vanishing activation barriers is relevant for our understanding of charge transport in organic semiconductors.
\end{abstract}




\section{Introduction}

Several theoretical frameworks have been developed to treat coupled electron-nuclear dynamics including multiconfigurational time-dependent Hartree, ${ }^{1,2}$ ab-initio multiple spawning, ${ }^{3,4}$ exact factorisation of the molecular wavefunction, ${ }^{5-7}$ path integral Monte Carlo, ${ }^{8-10}$ ring-polymer molecular dynamics ${ }^{11,12}$ and mixed quantumclassical nonadiabatic molecular dynamics (MQC-NAMD). ${ }^{7,13,14}$ Tully's fewest switches surface hopping (SH) method, ${ }^{14-17}$ which belongs to the the latter category, has proven particularly popular due to its simplicity and applicability to large molecules and condensed phase systems. In this method the electronic wavefunction is propagated according to the time-dependent electronic Schrödinger equation while the nuclei are propagated classically on a single electronic potential energy surface at a time. Feedback from the electronic to the classical degrees of freedom is incorporated by stochastic hops of the nuclei between the electronic surfaces.

While intuitive and relatively straightforward to implement, SH is not rigorously derived from first principles, although attempts have been recently made to derive the algorithm from a firmer physical footing. ${ }^{18}$ Hence, its suitability for and application to the specific question of electron transfer (ET) reactions has been investigated, but mostly for simple physical model systems such as spin-boson models. ${ }^{19-22}$ For example, Tully and co-workers and Sherman et al. have discussed whether or not the algorithm reproduces Boltzmann statistics with regard to the populations of the electronic states. ${ }^{19,20,23}$ Subotnik and co-workers have investigated the dependence of the SH ET rate on electronic coupling strength ${ }^{21}$ and driving force, ${ }^{21}$ as well as the effect of decoherence corrections on the ET rate. ${ }^{21,22}$

The performance of SH in describing electron transfer (ET) in real molecules and in condensed phase systems is less well tested. The problem is that exact results are no longer available for realistic systems and reliance must be placed on approximate but empirically validated theories, in this case semi-classical ET (Marcus) theory. ${ }^{24-28}$ The success of this theory in predicting rates for a wide range of ET reactions is undisputed, as is its simplicity and elegance. ${ }^{25}$ For ET reactions that are slow compared to the nuclear vibrations coupling to ET, this theory is expected to give good results and can be used to test $\mathrm{SH} \cdot{ }^{28}$ In the language of ET theory, this is the regime of large reorganisation energy $\lambda$ (electron-phonon coupling) and low transfer integral $H_{\mathrm{ab}}$ (diabatic electronic coupling) between initial and final electronic states.

Yet, derived from transition state theory ${ }^{28,29}$ and Landau-Zener theory for non-adiabatic electronic transitions, ${ }^{28,30,31}$ Marcus theory inherited the assumptions and approximations of those former theories making its applicability limited. In particular, for fast ET reactions (low $\lambda$ and/or high $H_{\mathrm{ab}}$ ), where the coupling between electronic and nuclear degrees of freedom is strong, the validity of Marcus theory is less clear. Here the separation of the full electron-nuclear problem in a nuclear (or Franck-Condon) factor and an electronic transmission coefficient (Landau-Zener) is problematic and it may no longer be safe to assume the validity of equilibrium statistical mechanics. NAMD methods that treat the coupling between electronic and nuclear degrees of freedom explicitly 
are expected to give a better description of fast ET and they may be used to test the results of Marcus theory in this regime.

In this contribution we report on $\mathrm{SH}$ simulations of ET for a simple donor-acceptor model complex in vacuum at full atomistic resolution using our recently developed fragment orbital-based surface hopping (FOB-SH) implementation with a simple correction for electronic decoherence. ${ }^{32}$ We investigate the rate dependence on the three parameters that the ET rate theory expressions depend upon: $\lambda, H_{\mathrm{ab}}$ and driving force $\Delta A$. The computed SH ET rates span a total of 4 orders of magnitude, from the sub-picosecond to the nanosecond time scale. For the slow regime $\left(H_{\mathrm{ab}}<<\lambda\right)$ we find that our results from $\mathrm{SH}$ agree to within a factor of 5 with ET rate theory (in most cases 2-3), while quantitative agreement appears to be at least partially dependent on the definition of ET rate in the SH simulations and the correct sampling of the equilibrium population of electronic states. In the limit of very large electronic coupling strength $\left(H_{\mathrm{ab}}>\lambda / 2\right)$ the free energy barrier disappears and we observe a crossover from ET to charge relaxation that is initially well described by thermally averaged Rabi-oscillations.

In the following section 2 we describe the FOB-SH methodology that we have implemented for SH simulation of ET reactions. We explain how ET rates are extracted from SH MD simulation data and summarise relevant rate expressions from ET theory. This is followed by a description of the molecular donor-acceptor model and the simulation details in section 3. The dependence of SH ET rates on reorganisation energy, electronic coupling strength and driving force will be presented and compared to the predictions of ET rate theory in section 4 . In section 5 we discuss the results obtained in view of some critical issues of the SH methodology: the definition of ET rates from SH MD simulation, the equilibrium population of electronic states and the decoherence correction.

\section{Theory}

\subsection{Fragment orbital-based surface hopping (FOB-SH)}

FOB-SH is a minimalistic but highly efficient implementation of Tully's fewest switches surface hopping for the simulation of charge transfer and transport in molecular and condensed phase systems. ${ }^{32}$ Here we summarise the most important features and the formulae that will be referred to in later sections. For a more detailed explanation of the method we refer to Ref. ${ }^{32}$

In FOB-SH it is assumed that the complicated many-body or multi-determinantal electron dynamics can be effectively described by a one-particle wavefunction $\Psi(t)$ for an excess charge (electron or electron hole) moving in an effective, time-dependent potential due to the other electrons and classical nuclear motion. The remaining valence and core electrons are not explicitly treated. Their interaction with the charge carrier is included implicitly through parametrisation of the electronic Hamiltonian (see below). We note that a similar strategy has been

employed previously for simulation of charge transfer in biomolecules. ${ }^{33,34}$ The charge carrier wavefunction is 
expanded in a basis of orthogonal fragment orbitals $\phi_{i}$ that mediate the charge transfer,

$$
\Psi(t)=\sum_{i=1}^{M} u_{i}(t) \phi_{i}(\mathbf{R}(t)),
$$

where $u_{i}(t)$ are the time-dependent expansion coefficients, $M$ the number of fragments or molecules participating in $\operatorname{ET~}(M=2$ for a donor-acceptor system with a single fragment orbital per site), and $\mathbf{R}$ the nuclear configuration vector. The fragment orbitals are obtained by orthogonalisation of the singly occupied molecular orbitals (SOMOs) of the molecules. Insertion of the expansion Eq. 1 in the time-dependent electronic Schrödinger equation gives

$$
i \hbar \dot{u}_{k}(t)=\sum_{j} u_{j}(t)\left(H_{k j}-i \hbar d_{k j}\right)
$$

where $H_{k j}$ are the elements of the electronic Hamiltonian $H$,

$$
H_{k j}=\left\langle\phi_{k}|H| \phi_{j}\right\rangle
$$

and $d_{k j}$ are the non-adiabatic coupling elements (NACEs),

$$
d_{k j}=\left\langle\phi_{k} \mid \dot{\phi}_{j}\right\rangle
$$

The diagonal elements of the electronic Hamiltonian, $H_{k k}$, are calculated using a classical force field. The offdiagonal elements, $H_{k j}$ are obtained from the overlap $S_{k j}$ between the (non-orthogonal) SOMOs on fragments $k$ and $j$,

$$
H_{k j}=C S_{k j}
$$

In previous work we have calibrated the constant $C$ against wavefunction-theory validated DFT calculations and found that the linear relation Eq. 5 gives an excellent approximation and is transferable to a variety of different $\pi$-conjugated systems. ${ }^{35}$ For calculation of the overlaps $S_{k j}$, the DFT SOMOs are expanded in a minimum Slatertype basis, which makes the calculation analytic and ultrafast. The NACEs in Eq. 4 are obtained similarly from SOMO overlaps in the finite difference approximation. For more details on the calculation of $H_{k j}$ and NACEs we refer to Refs. ${ }^{32,35}$

In $\mathrm{SH}$ the nuclear dynamics is propagated on one of the adiabatic electronic state potential energy surfaces, $E_{n}(\mathbf{R})$, which is obtained by diagonalisation of the Hamiltonian in the charge-localised basis, Eq. 3. Hops between adiabatic state surfaces, say $m \leftarrow l$, occur stochastically according to the probability $g_{m l},{ }^{14}$

$$
\begin{aligned}
g_{m l} & =\frac{b_{m l} \Delta t}{a_{l l}} \\
b_{m l} & =-2 \operatorname{Re}\left(a_{m l}^{*} d_{m l}\right) \\
a_{m l} & =c_{m} c_{l}^{*},
\end{aligned}
$$

where $\Delta t$ is the time step for integration of the nuclear forces, $a_{m l}$ the elements of the electronic density matrix, and $c_{n}$ the expansion coefficients of the carrier wavefunction $\Psi(t)$ in the adiabatic electronic state basis $\left\{\psi_{n}\right\} . \psi_{n}=$ 
$\sum_{k} U_{k n} \phi_{k}$ with $U$ the unitary transformation matrix that diagonalises $H$ in Eq. 3 , and $E_{n}$ are the corresponding eigenvalues. A further note regarding terminology. Although the charge localised states $\left\{\phi_{n}\right\}$ have finite nonadiabatic coupling vectors in contrast to pure diabatic states, we still refer to them as diabatic states for simplicity.

Here we investigate the dynamics of hole transfer from a donor to an acceptor $(M=2)$. $\Psi(t)$ is expanded in the two orthogonalised SOMOs mediating the transfer from donor to acceptor, see Figure 1 (A). These two charge localised or diabatic electronic states are denoted "a" and "b" in the following. The adiabatic electronic potential energy surfaces are then given by

$$
E_{0 / 1}(\mathbf{R})=\frac{H_{\mathrm{aa}}(\mathbf{R})+H_{\mathrm{bb}}(\mathbf{R})}{2} \pm \frac{1}{2} \sqrt{\Delta E^{2}(\mathbf{R})+4 H_{\mathrm{ab}}^{2}(\mathbf{R})}
$$

where $E_{0}$ takes the minus sign as the adiabatic ground state and vice versa for the excited adiabatic state, $E_{1}$, and $\Delta E$ is the diabatic energy gap (also termed site energy difference)

$$
\Delta E(\mathbf{R})=H_{\mathrm{bb}}(\mathbf{R})-H_{\mathrm{aa}}(\mathbf{R})
$$

The forces on each of these adiabatic surfaces is obtained by differentiation of Eq. 9,

$$
\mathbf{F}_{I, 0 / 1}=-\frac{\nabla_{I} H_{\mathrm{aa}}+\nabla_{I} H_{\mathrm{bb}}}{2} \mp \frac{\Delta E\left(\nabla_{I} H_{\mathrm{bb}}-\nabla_{I} H_{\mathrm{aa}}\right)+4 H_{\mathrm{ab}} \nabla_{I} H_{\mathrm{ab}}}{2 \sqrt{\Delta E^{2}+4 H_{\mathrm{ab}}^{2}}} .
$$

The corresponding force expression for a general $M$-site system is given in Ref. ${ }^{32}$ The gradients of the site energies, $\nabla_{I} H_{\mathrm{aa}}$ and $\nabla_{I} H_{\mathrm{bb}}$, are obtained from the force field. The only term in Eq. 11 that we do not already know is the gradient of the electronic coupling, $\nabla_{I} H_{\mathrm{ab}}$. Analogously to Eq. 5, we can use the approximation

$$
\nabla_{I} H_{\mathrm{ab}}=C \nabla_{I} S_{\mathrm{ab}}
$$

The $\nabla_{I} S_{\mathrm{ab}}$ can be expressed in terms of the non-adiabatic coupling vectors (NACVs) between the charge localised states, $\mathbf{d}_{I, \mathrm{ab}}^{\prime}$,

$$
\nabla_{I} S_{\mathrm{ab}}=\nabla_{I}\left\langle\varphi_{\mathrm{a}} \mid \varphi_{\mathrm{b}}\right\rangle=\left\langle\nabla_{I} \varphi_{\mathrm{a}} \mid \varphi_{\mathrm{b}}\right\rangle+\left\langle\varphi_{\mathrm{a}} \mid \nabla_{I} \varphi_{\mathrm{b}}\right\rangle=\mathbf{d}_{I, \mathrm{ba}}^{\prime *}+\mathbf{d}_{I, \mathrm{ab}}^{\prime}
$$

which we calculate in the finite difference approximation using the analytic overlap method described before.

\subsection{Electron transfer rates from $\mathrm{SH}$}

We have investigated two methods for the calculation of ET rates from SH based on two different observables. The first observable is the population decay of the initial diabatic state and the other the number of successful transitions between initial and final diabatic state per unit time. For a point charge transferring between donor and acceptor, the ET rates from population decay and transition count will give the same result. However, in $\mathrm{SH}$ the charge is a quantum mechanical object and may delocalise over donor and acceptor. In this case the two definitions are no longer expected to give the same results. The population decay rate is the natural choice when 
comparing to experimental population decay measurements, whereas the transition count rate is arguably better suited for comparison to ET (Marcus) theory as the latter gives the rate for transfer from initial to final diabatic state.

Population decay. In this method we monitor the amplitude of the initial diabatic state, $\left|u_{\mathrm{a}}\right|^{2}(t)$, and average over a large number of SH trajectories to obtain the electronic population

$$
P_{\mathrm{a}}(t)=\left\langle\left|u_{\mathrm{a}}\right|^{2}\right\rangle(t)
$$

As we will see later, the population decays obtained are to a good approximation exponential. Hence, a fit

$$
P_{\mathrm{a}}(t)=a \exp \left(-k_{\mathrm{d}} t\right)+b
$$

gives the population decay constant $k_{\mathrm{d}}$ (subscript "d") and $a, b$ are constant fit parameters. For a reversible reaction of the type studied here,

$$
\mathrm{A} \underset{k_{\mathrm{b}}}{\stackrel{k_{\mathrm{f}}}{\rightleftharpoons}} \mathrm{B} \text {, }
$$

the population decay of $\mathrm{A}$ is given by

$$
P_{\mathrm{A}}(t)=\frac{k_{\mathrm{f}}}{k_{\mathrm{f}}+k_{\mathrm{b}}}\left[\frac{k_{\mathrm{b}}}{k_{\mathrm{f}}}+\exp \left(-\left(k_{\mathrm{f}}+k_{\mathrm{b}}\right) t\right)\right]
$$

Comparing Eq. 17 with Eq. 15 and identifying $P_{\mathrm{A}}$ with $P_{\mathrm{a}}$, we find $k_{\mathrm{d}}=k_{\mathrm{f}}+k_{\mathrm{b}}$ and $a=k_{\mathrm{f}} / k_{\mathrm{d}}$. Inserting the definition of the equilibrium constant $K=k_{\mathrm{f}} / k_{\mathrm{b}}=\exp \left[-\Delta A /\left(k_{\mathrm{B}} T\right)\right]$, one obtains the SH ET rate from the population decay (subscript "p"),

$$
k_{\mathrm{p}}^{\mathrm{SH}} \equiv k_{f}=k_{\mathrm{d}}(1+\exp (\beta \Delta A))^{-1},
$$

as well as the driving force, $\Delta A=k_{\mathrm{B}} T \ln (1 / a-1)$.

Subotnik and co-workers have suggested two more definitions of diabatic state population $P_{\mathrm{a}}$, in addition to the one given in Eq. 14 which was denoted "Method 2" in their paper. ${ }^{36}$ In the surface-based method ("Method 1" in Ref. ${ }^{36}$ ) ET is measured by the time evolution of the projection of the active adiabatic electronic state $\lambda$ on the initial diabatic state. That is, the element of the matrix transforming between adiabatic and charge-localised states, $P_{\mathrm{a}}=\left\langle\left|U_{\mathrm{a} \lambda}\right|^{2}\right\rangle(t)$. In "Method 3" the mixed quantum-classical density is used to describe the population, $P_{\mathrm{a}}=\left\langle\sum_{i=0}^{1}\left|U_{\mathrm{a} i}\right|^{2}(t) \delta_{i, \lambda}\right\rangle+\left\langle\sum_{i<j} 2 \operatorname{Re}\left(U_{\mathrm{a} i}(t) \sigma_{i j}(t) U_{\mathrm{a} j}^{*}(t)\right)\right\rangle$, where $\sigma_{i j}=c_{i} c_{j}^{*}$. We will compare all three population decay methods in section 4 .

Transition count. Here we simply count the number of times, $N_{\mathrm{t}}$, that a full electron or electron hole (defined further below) transfers from the donor to the acceptor per second, and average over a large number of SH trajectories of length $T$,

$$
k_{\mathrm{t}}^{\mathrm{SH}}=\frac{1}{T}\left\langle N_{\mathrm{t}}\right\rangle
$$


where the subscript "t" refers to transition count. Being a quantum mechanical object, the charge is never fully located on either donor or acceptor (except at $t=0$ ). Hence we need to allow for some finite delocalisation and consider a transfer from donor to acceptor as successful when the amplitude changes from $\left|u_{\mathrm{a}}\right|^{2} \geq 0.9$ to $\left|u_{\mathrm{a}}\right|^{2} \leq 0.1$. These ranges were found to adequately describe the transitions in the system. While reasonable changes to these ranges do not significantly affect results, their definition is to some extent arbitrary, which needs to be taken into consideration when making quantitative comparison to Marcus theory.

\subsection{Electron transfer rate theory}

Consistent with the mixed quantum-classical SH approach of section 2.1, we consider here semi-classical ET theory where nuclei are treated as classical particles. Combining transition state theory (TST) in the harmonic approximation $^{28,29}$ and Landau-Zener theory (LZT) for the probability of electronic transitions between initial and final diabatic ET states, ${ }^{28,30,31}$ one obtains the following semi-classical (sc) expression for the rate of ET in the donor-acceptor complex, ${ }^{26-28,37}$

$$
k_{\mathrm{sc}}=\kappa_{\mathrm{el}} \nu_{\mathrm{n}} \exp \left(-\frac{\Delta A^{\ddagger}}{k_{\mathrm{B}} T}\right) .
$$

In Eq. 20, $\kappa_{\mathrm{el}}$ is the electronic transmission coefficient, $\nu_{n}$ is an effective nuclear frequency along the reaction coordinate, $\Delta A^{\ddagger}$ is the ET activation free energy, $k_{\mathrm{B}}$ the Boltzmann constant and $T$ the temperature. Equation 20 is sometimes augmented with a nuclear tunnelling factor that we set equal to unity here. The electronic transmission coefficient is given by $^{27}$

$$
\begin{aligned}
\kappa_{\mathrm{el}} & =\left\{\begin{array}{l}
\frac{2 P_{\mathrm{LZ}}}{1+P_{\mathrm{LZ}}} \quad \text { if } \quad \Delta A \geq-\lambda \\
2 P_{\mathrm{LZ}}\left(1-P_{\mathrm{LZ}}\right) \quad \text { if } \quad \Delta A<-\lambda
\end{array}\right. \\
P_{\mathrm{LZ}} & =1-\exp (-2 \pi \gamma) \\
2 \pi \gamma & =\frac{\pi^{3 / 2}\left\langle\left|H_{\mathrm{ab}}\right|^{2}\right\rangle_{\mathrm{TS}}}{h \nu_{\mathrm{n}} \sqrt{\lambda k_{\mathrm{B}} T}},
\end{aligned}
$$

where $P_{\mathrm{LZ}}$ is the Landau-Zener transition probability for a single crossing event from the initial to the final diabatic state surface, $\lambda$ is the reorganisation free energy, $h$ is Planck's constant, and $\left\langle\left|H_{\mathrm{ab}}\right|^{2}\right\rangle_{\mathrm{TS}}$ is the thermal average of the squared electronic coupling in the transition state (TS). We note that the TS is the union of configurations where the initial and final diabatic state surfaces cross, i.e. $\Delta E=0$. For definition of the activation free energy $\Delta A^{\ddagger}$, we consider the adiabatic ground state free energy profile along $\Delta E,^{38}$

$$
A_{0}(\Delta E) \simeq \frac{A_{\mathrm{a}}(\Delta E)+A_{\mathrm{b}}(\Delta E)}{2}-\frac{1}{2} \sqrt{\Delta E^{2}+4\left\langle\left|H_{\mathrm{ab}}\right|^{2}\right\rangle_{\Delta E}}
$$

where $A_{\mathrm{a}}(\Delta E)$ and $A_{\mathrm{b}}(\Delta E)$ are the Marcus parabola for initial and final diabatic states. ${ }^{38}$ We take the ET activation free energy as the difference in free energy at the TS $(\Delta E=0)$ and at the minimum of the adiabatic 
free energy profile corresponding to the initial state $\left(\Delta E=\Delta E_{0}\right)$,

$$
\begin{aligned}
\Delta A^{\ddagger} & =A_{0}(\Delta E=0)-A_{0}\left(\Delta E=\Delta E_{0}\right) \\
& =\Delta A_{\text {na }}^{\ddagger}-\Delta^{\ddagger} .
\end{aligned}
$$

$\Delta A_{\text {na }}^{\ddagger}$ is the activation free energy on the diabatic electronic states,

$$
\Delta A_{\mathrm{na}}^{\ddagger}=\frac{(\lambda+\Delta A)^{2}}{4 \lambda}
$$

and $\Delta^{\ddagger}$ is given by

$$
\Delta^{\ddagger}=\left\langle\left|H_{\mathrm{ab}}\right|^{2}\right\rangle_{\mathrm{TS}}^{1 / 2}-\frac{1}{\lambda}\left\langle\left|H_{\mathrm{ab}}\right|^{2}\right\rangle_{\Delta E_{0}}
$$

for $\Delta A=0 . \Delta^{\ddagger}$ is a correction to $\Delta A_{\text {na }}^{\ddagger}$ that becomes important when electronic coupling is large, i.e. for ET in the adiabatic limit. The first term on the right hand side of Eq. 28 is the difference between adiabatic and diabatic free energy surface at the TS $(\Delta E=0)$ and the second term is the corresponding free energy difference at the minimum of the initial well, located at $\Delta E=\Delta E_{0}=-\left(\lambda^{2}-4\left\langle\left|H_{\mathrm{ab}}\right|^{2}\right\rangle_{\Delta E_{0}}\right)^{1 / 2}$ for $\Delta A=0$. Disregarding in the following the dependence of electronic electronic coupling on the reaction coordinate, $\left\langle\left|H_{\mathrm{ab}}\right|^{2}\right\rangle \equiv\left\langle\left|H_{\mathrm{ab}}\right|^{2}\right\rangle_{\mathrm{TS}}=\left\langle\left|H_{\mathrm{ab}}\right|^{2}\right\rangle_{\Delta E_{0}}$ and inserting Eqs. 28 and 27 in Eq. 26, we find that $\Delta A^{\ddagger}=0$ for $\left\langle\left|H_{\mathrm{ab}}\right|^{2}\right\rangle^{1 / 2}=\lambda / 2$, i.e. the barrier vanishes. For even larger values for electronic coupling the activation barrier would become negative. Therefore the rate Eq. 20 is only defined for $\left\langle\left|H_{\mathrm{ab}}\right|^{2}\right\rangle^{1 / 2} \leq \lambda / 2$. In previous works a different correction to $\Delta A_{\text {na }}^{\ddagger}$ was used, denoted $\Delta$ and obtained by assuming that $\Delta E_{0}$ coincides with the position of the minimum of the initial diabatic state $\Delta E_{a} \cdot{ }^{38-42}$ This is an approximation and should be replaced by Eq. 28 if $\Delta A=0$ (an expression for $\Delta^{\ddagger}$ for general $\Delta A$ is rather lengthy).

Equation 20 describes ET in the non-adiabatic, adiabatic and intermediate regimes. These regimes are defined by the adiabaticity parameter $2 \pi \gamma$, Eq. 23. If $2 \pi \gamma<<1$ the reaction is non-adiabatic (na) and the exponent in Eq. 22 can be expanded in a Taylor series and truncated after the first order term, giving $P_{\mathrm{LZ}}=2 \pi \gamma$ and $\kappa_{\mathrm{el}}=2 P_{\mathrm{LZ}}$. Insertion of this result into Eq. 20 gives the non-adiabatic ET rate, ${ }^{25,28}$

$$
k_{\mathrm{na}}=\frac{2 \pi}{\hbar}\left\langle\left|H_{\mathrm{ab}}\right|^{2}\right\rangle_{\mathrm{TS}}\left(4 \pi \lambda k_{\mathrm{B}} T\right)^{-1 / 2} \exp \left(-\frac{\Delta A_{\mathrm{na}}^{\ddagger}}{k_{\mathrm{B}} T}\right) .
$$

In the opposite limit $2 \pi \gamma>>1$ the ET is adiabatic (ad) and $P_{\mathrm{LZ}}$ and $\kappa_{\mathrm{el}}$ approach unity. The rate expression is the same as in classical transition state theory for chemical reactions that occur on a single adiabatic potential energy surface. The prefactor depends only on the frequency along the reaction coordinate, ${ }^{28}$

$$
k_{\mathrm{ad}}=\nu_{\mathrm{n}} \exp \left(-\frac{\Delta A^{\ddagger}}{k_{\mathrm{B}} T}\right) .
$$




\section{Simulation details}

\subsection{Molecular model}

We have chosen to carry out simulations for electron hole transfer between two ethylene-like molecules (ELMs), see Figure 1 (A). We refer to them as "ethylene-like" because while the atomic geometries correspond to a physical ethylene molecule, we have chosen to parametrise them to reproduce arbitrary values of the reorganisation energy $\lambda$, average electronic coupling $\left\langle\left|H_{\mathrm{ab}}\right|\right\rangle$ and driving force $\Delta A$. The molecular parametrisation described below is rather general and can be carried out for any pi-conjugated organic molecule. The site energies $H_{k k}(k=\mathrm{a}, \mathrm{b})$ and corresponding forces $\nabla_{I} H_{k k}$ are calculated with a classical force field. For the neutral ELM we use the standard parameters for ethylene from the Generalised Amber Force Field (GAFF). ${ }^{43}$ For the positively charged ELM, the excess charge of +1 is equally distributed over the two $\mathrm{C}$ atoms. The $\mathrm{C}=\mathrm{C}$ equilibrium bond length of the charged ELM, $r_{0}$, was adjusted to obtain a given value of reorganisation energy $\lambda$,

$$
\lambda=\left[E_{\mathrm{C}}\left(\mathbf{R}_{\mathrm{N}}\right)+E_{\mathrm{N}}\left(\mathbf{R}_{\mathrm{C}}\right)\right]-\left[E_{\mathrm{C}}\left(\mathbf{R}_{\mathrm{C}}\right)+E_{\mathrm{N}}\left(\mathbf{R}_{\mathrm{N}}\right)\right] .
$$

All other parameters are the same as for the neutral ELM, including the force constant of the $\mathrm{C}=\mathrm{C}$ bond, 589.7 kcal mol ${ }^{-1} \AA^{-2}$. In Eq. 31, $E_{\mathrm{C}}\left(\mathbf{R}_{\mathrm{N}}\right)$ is the total force field energy of the charged ELM (subscript C) at the minimum energy configuration of the neutral ELM, $\mathbf{R}_{\mathrm{N}}, E_{\mathrm{N}}\left(\mathbf{R}_{\mathrm{C}}\right)$ is the total force field energy of the neutral ELM (subscript $\mathrm{N}$ ) at the minimum energy configuration of the charged ELM, $\mathbf{R}_{\mathrm{C}}$, and $E_{\mathrm{C}}\left(\mathbf{R}_{\mathrm{C}}\right), E_{\mathrm{N}}\left(\mathbf{R}_{\mathrm{N}}\right)$ are the energies at the minimum energy configurations of the respective states. Our default value is $\lambda=0.3 \mathrm{eV}$, unless noted otherwise. The values of $\lambda$ and $r_{0}$ used for investigation of the ET rate dependence on $\lambda$ are summarised in Table 1. The driving force $\Delta A$ between the two ELMs is by default zero (hole self-exchange). Simulations at finite driving forces are modelled by simply adding a constant shift of $\Delta A$ to $H_{\mathrm{bb}}$. Electronic coupling matrix elements, $H_{\mathrm{ab}}$, are calculated using the $\mathrm{AOM}$ method, Eq. 5, with $C$ treated as a parameter controlling the strength of electronic coupling. The default value is $C=89 \mathrm{meV}$ giving $\left\langle\left|H_{\mathrm{ab}}\right|^{2}\right\rangle^{1 / 2}=8 \mathrm{meV}$, unless noted otherwise. The values for $C$ and the resulting values of electronic coupling used for investigation of the ET rate dependence on the coupling strength are summarised in Table 2 . The calculation of the overlap integrals $S_{k l}$ was carried out as described in Ref. ${ }^{35}$ using a minimum Slater basis of $p$ orbitals with Slater decay coefficients $\bar{\mu}_{2 p}=1.0000$ a.u. ${ }^{-1}$. The NACVs, and by virtue of Eqs. $12-13 \nabla_{I} H_{k l}$ and $\nabla_{I} S_{k l}$, respectively, are obtained by numeric differentiation using an increment for nuclear displacements of $\Delta s=10^{-4} \AA$. For calculation of the hopping probability Eq. 6, the NACEs are calculated by numeric differentiation using a time increment $\Delta t=0.5$ fs.

\subsection{FOB-SH simulations}

Initial configurations for FOB-SH were generated from classical MD simulation of the ELM dimer on the initial diabatic state potential energy surface $H_{\text {aa }}$, i.e. with the hole fully localised on ELM 1 . The dimer was initially 
placed in a stacked configuration at a distance of $4 \AA$. A weak harmonic position restraint with force constant of $1.0 \mathrm{kcal} \mathrm{mol} \mathrm{m}^{-1} \AA^{-2}$ was applied to each carbon atom to maintain a stable intermolecular separation while still allowing for rotational motion of the monomers. FOB-SH simulations at zero driving force $(\Delta A=0)$ are initiated from structures taken from the classical MD trajectory and propagated on the adiabatic ground state $E_{0}$, noting that $H_{\mathrm{aa}} \approx E_{0}$ in the initial state diabatic well. For increasingly negative driving forces the initial state becomes unstable against thermal fluctuations as the avoided crossing moves towards the initial state minimum. Here FOB-SH simulations are initiated from $E_{0}$ when the energy gap $\Delta E \geq 0$ and from the excited state $E_{1}$ otherwise. For strongly negative driving forces deep in the Marcus inverted regime, $H_{\mathrm{aa}} \approx E_{1}$. Hence FOB-SH simulations are initiated from $E_{1}$ in this regime. Consistently with this, the initial conditions for hole propagation according to Eq. 2 are $u_{\mathrm{a}}(0)=1, u_{\mathrm{b}}(0)=\dot{u}_{\mathrm{a}}(0)=\dot{u}_{\mathrm{b}}(0)=0$. Equation 2 for the charge carrier dynamics and Newton's equation of motion for the nuclear dynamics are integrated numerically using an electronic time step $\delta t=0.1 \mathrm{fs}$ and a nuclear time step $\delta t=0.5 \mathrm{fs}$, respectively. Surface hops are attempted every nuclear time step and the velocities are rescaled after each successful hop to conserve total energy as explained in Ref. ${ }^{32}$ The simulations are all carried out in the NVT ensemble, with a Langevin thermostat applied at $300 \mathrm{~K}$ with a friction constant of $\gamma=10 \mathrm{ps}^{-1}$. The energy conservation of our FOB-SH implementation was investigated and discussed previously for the same ELM dimer system. ${ }^{32}$ The energy drift in the NVE ensemble is reasonably small, in the order of $10^{-6}$ Hartree atom ${ }^{-1} \mathrm{ps}^{-1}$. This is larger than in classical MD simulations using the same MD code $\left(\approx 10^{-9}\right.$ Hartree atom $\left.{ }^{-1} \mathrm{ps}^{-1}\right)$ due to the discontinuity of forces after a surface hop. The problem of electronic over-coherence in $\mathrm{SH}^{21,22,44-50}$ is accounted for similarly as in Ref. ${ }^{21,45}$ After a surface hop from the excited to the adiabatic ground state we collapse the electronic wavefunction onto the adiabatic ground state the first time that the system passes through one of the ground state minima while moving away from the avoided crossing. ${ }^{45}$ This is a simple and intuitive way to ensure that the wavefunction collapse only happens when the non-adiabatic coupling between the two states is negligible.

\subsection{ET rates}

ET rates from SH are obtained according to Eqs. 18 and 19 by averaging over 500 FOB-SH runs of variable length in the range 2-500 ps depending on the decay rate. The population decays at zero driving force were found to fit well to a single exponential, Eq. 15, with $R^{2}$ values of at least 0.92 . For calculation of rates from ET theory, Eqs. 20, 29 and 30, we obtained values for $\left\langle\left|H_{\mathrm{ab}}\right|^{2}\right\rangle_{\mathrm{TS}}, \lambda, \Delta A$ and $\nu_{\mathrm{n}}$ as follows. $H_{\mathrm{ab}}$ was averaged over the MD trajectories used to initialise the FOB-SH simulations. This gives the average for the initial diabatic state, $\left\langle\left|H_{\mathrm{ab}}\right|^{2}\right\rangle_{\mathrm{a}}$. The difference with respect to the ensemble average at the TS is expected to be very small and was neglected. Reorganisation energies $\lambda$ were also obtained from these MD runs by averaging over the energy gap, $\lambda=\langle\Delta E\rangle_{\mathrm{a}}$. The numerical values were found to be virtually identical with the ones defined in Eq. 31 for $0 \mathrm{~K}$ 
at infinite donor-acceptor separation. For $\Delta A$ we used the same numerical values as applied in SH simulation. The frequency along the reaction coordinate, $\nu_{n}$, was obtained by calculating the spectral density function of the energy gap as obtained from the above MD simulations in the initial state. ${ }^{38}$ The spectrum features a main peak at $\nu_{n}=1700 \mathrm{~cm}^{-1}$, representative of the $\mathrm{C}=\mathrm{C}$ stretch vibration.

\section{Results}

Before we present our main findings we briefly illustrate the system and its behaviour in Fig. 1. In panel $\mathrm{A}$ is a pictorial representation of the simulated ELM donor-acceptor system with the excess hole (SOMO) transferring from donor to acceptor. Some properties along a typical FOB-SH run for this system $\left(\lambda=0.3 \mathrm{eV},\left\langle\left|H_{\mathrm{ab}}\right|^{2}\right\rangle^{1 / 2}=\right.$ $8 \mathrm{meV}$ and $\Delta A=0)$ are shown in panels B-D: the amplitude of the initial state, $\left|u_{\mathrm{a}}\right|^{2}$, the time series of the energy gap $\Delta E$, Eq. 10, and the active adiabatic electronic state used to propagate the nuclear dynamics. We can see that the system begins with the hole localised on the donor, with the site energy difference $\Delta E$ oscillating around the corresponding ground state minimum $(\Delta E \approx \lambda=0.3 \mathrm{eV})$. As thermal oscillations bring $\Delta E$ close to 0 , surface hops to the excited state and back occur and at the same time charge begins to transfer across to the acceptor, until eventually the system settles down on the ground state with the excess charge now localised on the acceptor. In this example, the same process later occurs in reverse, returning the system to its initial state.

This example trajectory in Fig. 1 gives us some idea of what to qualitatively expect from the rate dependencies. It is clear that large-scale charge transfer requires the system to be close to the crossing point at $\Delta E=0$. For larger values of $\lambda$, the activation barrier will be higher and the initial state will be further from the crossing point, so we expect the rate to decrease with increased $\lambda$. Conversely, as $H_{\mathrm{ab}}$ increases the barrier height will decrease, so the system will reach the crossing region more easily. In addition, the frequency of the intrinsic electron dynamics (Rabi-type oscillations) will increase possibly further contributing to an increase in rate with increasing $H_{\mathrm{ab}}$. Both of these behaviours are also predicted by ET theory, suggesting our SH simulations can (at least) qualitatively reproduce standard results.

SH rates have been calculated by both the transition count method and by an exponential fit to the population decay of the initial state, as described in section 3. In Ref. ${ }^{36}$ three possible definitions of the electronic population $P_{\mathrm{a}}$ were proposed. For comparison, we have calculated and plotted all three for one set of $500 \mathrm{SH}$ trajectories, along with their respective exponential fits $\left(\lambda=0.1 \mathrm{eV},\left\langle\left|H_{\mathrm{ab}}\right|^{2}\right\rangle^{1 / 2}=8 \mathrm{meV}\right.$ and $\left.\Delta A=0\right)$. As one can see in Fig. 2, the results are very similar for all three methods with ET rates falling in a narrow range of $0.75-1.14 \times 10^{12} \mathrm{~s}^{-1}$ (black lines). Moreover, we find that the decoherence correction leads to a slight decrease in the ET rate to $1.05 \times 10^{12} \mathrm{~s}^{-1}$ (red solid line) from $1.14 \times 10^{12} \mathrm{~s}^{-1}$ (black dashed line), improving the agreement with the rate from ET theory, $0.88 \times 10^{12} \mathrm{~s}^{-1}$. All of the following results have been obtained from $\mathrm{SH}$ simulations with decoherence correction and from exponential fits to the population decay of the initial electronic state according to Eq. 14 (method 2). 


\section{$4.1 \lambda$ dependence}

In Fig. 3 we plot ET rates from $\mathrm{SH}$ transition count $\left(k_{\mathrm{t}}^{\mathrm{SH}}\right.$, diamonds) and population decay $\left(k_{\mathrm{p}}^{\mathrm{SH}}\right.$, circles $)$ against reorganisation energy $\lambda$. We compare these against results from semi-classical ET theory, Eq. 20 (solid lines). We immediately observe a good agreement between the transition count rates and ET theory, with absolute values differing by less than a factor of 3 . Even for the lowest value of $\lambda$, where the activation barrier is on the order of $k_{\mathrm{B}} T$ and the ET is very fast, this excellent agreement consists. The population decay rates are also in good agreement with ET theory for small $\lambda$ values, but there are significant deviations of up to a factor of 5 for larger $\lambda$ values. Both the SH transition count and exponential decay rates appear to predict similar slopes for rate vs. $\lambda$, and these slopes are also slightly shallower than those predicted by ET theory. In the following we try to rationalise these findings.

Seeking to analyse at first the good agreement between ET theory and SH transition count rates, we consider the ET rate $k_{\mathrm{sc}}$ in Eq. 20 as a product of the rate in the adiabatic limit, $k_{\mathrm{ad}}$ (Eq. 30), which is a purely nuclear rate factor, and the electronic transmission coefficient $\chi_{\mathrm{el}}, k_{\mathrm{sc}}=\chi_{\mathrm{el}} k_{\mathrm{ad}}$. Similarly, $k_{\mathrm{t}}^{\mathrm{SH}}$ is considered as a product of a purely nuclear rate obtained by counting nuclear crossings during SH simulation from initial to final state well, $k_{\mathrm{t}, \mathrm{ad}}^{\mathrm{SH}}$, and a corresponding electronic transmission coefficient $\chi_{\mathrm{t}, \mathrm{el}}^{\mathrm{SH}}=k_{\mathrm{t}}^{\mathrm{SH}} / k_{\mathrm{t}, \mathrm{ad}}^{\mathrm{SH}}$. We can see in Fig. 3 that $k_{\mathrm{t}, \mathrm{ad}}^{\mathrm{SH}}$ (plus symbols) is smaller than $k_{\mathrm{ad}}$ (dashed lines), by a factor of up to about 5 . This can be attributed to frictional effects on the molecules, leading to unsuccessful crossings and recrossings at the barrier top, not taken into account by ET theory. Conversely, the electronic transmission coefficient in SH is by about the same amount larger than what is expected according to ET theory, more specifically Landau-Zener theory. Those two opposing effects largely cancel one another and lead to similar overall ET rates.

The increasing difference between the transition count $\left(k_{\mathrm{t}}^{\mathrm{SH}}\right)$ and population decay ET rates $\left(k_{\mathrm{p}}^{\mathrm{SH}}\right)$ with increasing $\lambda$ can be understood by analysing the qualitative behaviour of the donor amplitude $\left|u_{\mathrm{a}}\right|^{2}(t)$. In Fig. 4 we show $\left|u_{\mathrm{a}}\right|^{2}(t)$ for small $\lambda(0.1 \mathrm{eV}$, panel A) and for large $\lambda(0.7 \mathrm{eV}$, panel $\mathrm{B})$. At $0.1 \mathrm{eV}$ the barrier on the ground state is about $k_{\mathrm{B}} T$. Barrier crossings, switches to the excited state and generation of resonant donor-acceptor structures (defined by $-H_{\mathrm{ab}} \leq \Delta E \leq H_{\mathrm{ab}}$ ) occur very frequently, resulting in rapid, Rabi-like oscillations of one full hole between donor and acceptor. Our chosen threshold for a successful transfer is indicated by dashed lines. At $0.7 \mathrm{eV}$ (panel B) the barrier increases and becomes more steep. Resonance occurs infrequently and for shorter durations. This can lead to "interruption" of hole transfer by the system moving quickly away from the crossing point before the hole becomes fully localised on a single site. The decoherence correction then causes $\left|u_{\mathrm{a}}\right|^{2}$ to attain values close to 0 or 1 , depending on whether the system settles in the initial or final well. While each interrupted transfer event contributes to the population decay and hence to $k_{\mathrm{p}}^{\mathrm{SH}}$, only the fraction of events that settle in the final well count towards $k_{\mathrm{t}}^{\mathrm{SH}}$. This explains the consistently higher $k_{\mathrm{p}}^{\mathrm{SH}}$ compared with $k_{\mathrm{t}}^{\mathrm{SH}}$. 


\section{$4.2 \quad H_{\mathrm{ab}}$ dependence}

The dependence of ET rates on average electronic coupling strength, $\left\langle\left|H_{\mathrm{ab}}\right|^{2}\right\rangle^{1 / 2}$, is shown in Fig. 5 A. We

plot the $\mathrm{SH}$ population decay rate $\left(k_{\mathrm{p}}^{\mathrm{SH}}\right.$, circles) alongside results from ET theory (lines) - in this instance we plot the semi-classical ET rate Eq. 20 as well as both the non-adiabatic and adiabatic ET rates, Eqs. 29 and 30 respectively, to demonstrate how the former interpolates between the latter two expressions. In panel (B) we show the population decay for selected points marked by vertical arrows in panel $\mathrm{A}$, and panel $\mathrm{C}$ displays the corresponding free energy profiles $A_{0}$, Eq. 24 .

The agreement between $k_{\mathrm{p}}^{\mathrm{SH}}$ and ET theory (Eq. 20) is particularly good in the small coupling regime $(0-$ $30 \mathrm{meV} \leq \lambda / 10$ ) with deviations of less than a factor of about 2 . We fit a straight line $\log k_{\mathrm{p}}^{\mathrm{SH}}=a \log \left\langle\left|H_{\mathrm{ab}}\right|^{2}\right\rangle^{1 / 2}+b$ to the points in this range and obtain $a=2.0$, in good agreement with the prediction for the non-adiabatic limit Eq. 29, for which $a=2$. For larger coupling values $(\geq 30 \mathrm{meV}=\lambda / 10)$ the ET becomes increasingly adiabatic and the SH ET rates fall slightly below the semi-classical ET rate. In this regime the barrier is only about $k_{\mathrm{B}} T$ in our simulations (red line in panel $\mathrm{C}$ ), and we observe that fits to a mono-exponential decay are no longer as good as for the non-adiabatic regime (data in red in panel B). This is probably the reason for the deviation with semiclassical ET theory, which assumes exponential decay. Transition count ET rates (not shown) give similar results as the population decay SH ET rates discussed here. The former rates are again somewhat lower than the latter, which we ascribe to the same reason as in Fig. 3.

An interesting situation occurs when $\left\langle\left|H_{\mathrm{ab}}\right|^{2}\right\rangle^{1 / 2}=\lambda / 2(150 \mathrm{meV})$. At this point the barrier for ET completely disappears (see Eq. 26, purple line in panel (C)) and we observe charge relaxation of the initially localised hole to a fully delocalised hole on the fs time scale. This is characterised by a crossover from exponential to damped oscillatory population decay (purple circles, inset panel (B)). At short times (first few fs), the damped oscillation is well described by a thermally averaged Rabi-oscillation (dashed lines, inset panel (B)),

$$
P_{\mathrm{a}}(t)=\left\langle 1-\frac{4\left|H_{\mathrm{ab}}\right|^{2}}{\Delta E^{2}+4\left|H_{\mathrm{ab}}\right|^{2}} \sin ^{2}\left(\frac{1}{2} \Omega_{\mathrm{R}} t\right)\right\rangle_{E_{\mathrm{a}}}
$$

where $\Omega_{\mathrm{R}}$ is the Rabi frequency, given by

$$
\Omega_{\mathrm{R}}=\frac{1}{\hbar} \sqrt{\Delta E^{2}+4 H_{\mathrm{ab}}^{2}}
$$

In Eq. 32 the average is taken over the ensemble of initial diabatic state configurations from which SH MD runs were initiated. We define a relaxation rate $1 / t_{\max }$ for this process, where $t_{\max }$ is the time at which the damped oscillation reaches its first maximum. We find that the the relaxation rate increases approximately linearly with electronic coupling strength, as shown in panel A (diamonds). This is in agreement with the Rabi frequency increasing linearly with increasing electronic coupling $\left(\Omega_{\mathrm{R}}=2 H_{\mathrm{ab}} / \hbar\right.$ at $\left.\Delta E=0\right)$. The population decay becomes more complicated at longer times when nuclear motion leads to fluctuations in $\Delta E$. 


\section{3 $\Delta A$ dependence}

Finally we investigate whether SH can reproduce the characteristic inverse power-law dependence of ET rates on driving force and the Marcus inverted region in particular. In Fig. 6 we plot the SH rate obtained from the population decay $\left(k_{\mathrm{p}}^{\mathrm{SH}}\right.$, circles) alongside with the ET theory rate Eq. 20 (lines). The driving force is varied from $\Delta A=0$ to more negative values well beyond the onset of the inverted regime at $\Delta A=-\lambda$. The reaction now becomes biased towards the final state and is in fact irreversible on the time scale of present simulations. Once the hole is localised on the acceptor we do not observe the reverse transfer. Therefore, the transition count method is no longer used for calculating rates. In Fig. 6 we see that $\mathrm{SH}$ is capable of reproducing the inverse power law of ET theory in broad strokes. A fit $\log k_{\mathrm{p}}^{\mathrm{SH}}=-(\Delta A+a)^{2} /\left(4 b k_{\mathrm{B}} T\right)+c\left(R^{2}=0.64\right)$ gives $a=282 \mathrm{meV}, b=410$ $\mathrm{meV}$, which should be compared to the result of Marcus theory, $a=b=\lambda=0.3 \mathrm{eV}$. Some data points do not join the others, in particular around the maximum. We currently cannot provide an explanation for this, but note that the region around the maximum is a rather subtle regime, with some trajectories initiated in the ground and others in the excited state, and associated challenges in numerically converging results.

\section{Discussion}

We found that FOB-SH can reproduce very well the predictions of semi-classical ET theory over the time scales accessible to current simulations, from picosecond to nanosecond electron transfer. The qualitative trends for rate versus reorganisation energy, electronic coupling and driving force are correctly predicted with deviations that are generally less than half an order of magnitude with respect to ET theory. A more quantitative agreement depends on a number of issues that we would like to discuss in the following.

- Definition of ET rate from SH MD. As mentioned in section 2.2, there is a question over exactly how ET rates should be calculated from SH simulations. Extracting a rate from an exponential fit to $\left|u_{\mathrm{a}}\right|^{2}$ or another diabatic state quantity ${ }^{18}$ is analogous to experimental measurements of initial state population decay and, it can be argued, may not be directly comparable to e.g. Marcus theory. This is because the population decay can occur not only via transfer of a full charge from donor to a acceptor but also via delocalisation of the charge over donor and acceptor. ET theories account only for the former decay channel as they directly calculate a rate for transfer from the initial to the final diabatic state; they do not consider the possibility of charge delocalisation. In the extreme case, if an initial state amplitude $\left(\left|u_{\mathrm{a}}\right|^{2}\right)$ decays exponentially from 1.0 to 0.5 without ever showing values below 0.5 , the rate according to the population decay would be finite, whereas the semi-classical ET rate would be zero.

For this reason we have also investigated a transition count method that simply counts the number of successful transitions of a full charge from donor to acceptor per unit of time. The ET rates obtained in 
this way should correspond more closely to the ones from ET theory. However the results depend on the choice for the transition 'threshold' (chosen in our simulations as $\left|u_{\mathrm{a}}\right|^{2}$ dipping below 0.1): the most stringent definition possible $\left(\left|u_{\mathrm{a}}\right|^{2}\right.$ reaching 0) is likely to give us spurious rates, as the quantum oscillations of the electronic populations make it unlikely for this point to actually be reached; and without a clear justification for any other particular choice, a quantitative comparison between transition count rates and ET theory should also be treated with caution. The fact that we can calculate these rates in two different methods, each corresponding to a different experimental observable, suggests that our method could eventually be useful for comparison to a wide range of experimental results.

- Electronic state equilibrium population. Reviewing our results for the dependence of ET rate vs reorganisation energy $\lambda$, we notice that the SH ET rate (circles in Fig. 3) becomes overestimated with increasing $\lambda$. Hence, the slope expected from non-adiabatic ET theory, $\ln k_{\mathrm{ET}} \propto\left(k_{\mathrm{B}} T\right)^{-1} \lambda$ is somewhat underestimated, as if the temperature during the SH MD run would be higher than the actual simulation temperature $(300 \mathrm{~K})$. We think the reason for this is that the equilibrium population of the excited electronic state does not scale correctly with increasing reorganisation free energy in our SH MD runs. With increasing $\lambda$ the system spends too much time in the excited state resulting in too high ET rates. The effect is not dramatic (half on order of magnitude error at $\lambda=0.7 \mathrm{eV}$ ), but still significant. Part of this discrepancy is due to the fact that in this first version of FOB-SH we allow a surface hop to happen if the total kinetic energy is sufficient to compensate for the change in potential energy rather than the part of the kinetic energy that comes from the velocity component parallel to the NACVs. Therefore the number of frustrated hops to the excited state is too low in our simulations. In addition to this, recent analyses of the $\mathrm{SH}$ method for a two-level ${ }^{20}$ and three-level model system ${ }^{23}$ have shown that $\mathrm{SH}$ does not in general yield a Boltzmann equilibrium population of the electronic states. However, in practice the observed deviations are small, especially in the limits of small electronic coupling $\left(H_{\mathrm{ab}}\right)$ and/or strong nonadiabatic coupling $\left(\mathbf{d}_{k l}\right)$. Implementation of NACVs for adiabatic electronic states and improved treatment of classically forbidden electronic transitions ${ }^{51}$ in our FOB-SH method are likely to rectify this problem.

- Decoherence correction. The decoherence correction to SH, specifically the choice of how to correct for electronic over-coherence, has been widely discussed in the literature, and there is agreement that such a correction is vital to reproduce physical behaviour. ${ }^{21,22,44-50}$ Fig. 2 does suggest that this correction alters the ET rates that we calculate from SH simulation. However, the effect is not very strong. Thus far, the correction we have investigated is a very simple one, simply collapsing the wavefunction at certain points during the trajectory, and it is equally possible that there could be an effect on the rate from choosing different corrections for the over-coherence. This is something that could be investigated with future uses of our FOB-SH implementation. 
A second focus of our FOB-SH investigations was to probe the regime of large electronic couplings $(>\approx 50$ $\mathrm{meV}$ ) and small activation free energies $\left(\approx k_{\mathrm{B}} T\right)$, relevant to ET between $\pi$-conjugated molecules forming organic semiconductors. We find that non-adiabatic ET theory gives virtually identical rates as SH MD (grey region in Fig. 5), which should be considered fortuitous as non-adiabatic ET theory no longer applies in this regime. Interestingly, the more appropriate adiabatic ET theory does not describe the SH ET rates as well. Most likely, this is due to first signatures of non-exponential decay in the initial state population obtained from SH MD. At the point where the activation barrier vanishes $\left(H_{\mathrm{ab}}=\lambda / 2\right)$ we observe a crossover from fast ET on the vibronic time scale to ultrafast charge relaxation on the femtosecond time scale. Interestingly, if we extrapolate the non-adiabatic ET rate to this regime we obtain numerical estimates close to the charge relaxation rate. Hence, non-adiabatic ET theory and SH give rather similar rates across the entire spectrum of electronic coupling strengths. This observation may explain why electron mobilities in organic semiconductors could be reasonably well predicted with charge hopping models based on non-adiabatic ET theory, even though the underlying charge transfer process is likely to have little in common with non-adiabatic ET. ${ }^{39,40,52,53}$

Lastly, we conclude that the results reported are promising for future applications of the FOB-SH method to systems where analytic theories are not readily available. These are (i) the ultrafast ET regime (high electronic coupling/low reorganisation energy), (ii) systems where activation barriers can become time-dependent and the dynamics non-exponential and/or non-ergodic ${ }^{54}$ and (iii) large condensed phase systems with many electronically coupled redox active sites. Examples of such systems that are of interest to the scientific community include charge transport in strongly fluctuating structures in solid and solution phases such as organic semiconducting materials and redox active biomolecules.

\section{Acknowledgements}

J.S. was supported by an IMPACT PhD studentship co-sponsored by University College London and the Department of Physics and Astronomy. L. S. acknowledges ENS and A. C. EPSRC (Grant EP/M001989/1) for financial support. The authors acknowledge the use of the UCL High Performance Computing Facility "Legion", and associated support services.

\section{References}

[1] G. A. Worth, H.-D. Meyer, H. Koppel, L. S. Cederbaum, and I. Burghardt, Int. Rev. Phys. Chem., 2008, 27, 569-606.

[2] H. Tamura and I. Burghardt, J. Am. Chem. Soc., 2013, 135, 16364-16367.

[3] T. J. Martinez, M. Ben-Nun, and R. Levine, J. Chem. Phys., 1996, 100, 7884. 
[4] T. J. Martinez, Acc. Chem. Res., 2006, 39, 119-126.

[5] A. Abedi, N. T. Maitra, and E. K. U. Gross, Phys. Rev. Lett., 2010, 105, 123002.

[6] A. Abedi, N. T. Maitra, and E. K. U. Gross, J. Chem. Phys., 2012, 137, 22 A530.

[7] F. Agostini, A. Abedi, and E. K. U. Gross, J. Chem. Phys., 2014, 141, 214101.

[8] R. P. Feynman and A. R. Hibbs, Quantum Mechanics and Path Integrals, McGraw-Hill, New York, 1965.

[9] A. Kuki and P. Wolynes, Science, 1987, 236, 1647-1652.

[10] M. Marchi and D. Chandler, J. Chem. Phys., 1991, 95, 889-894.

[11] S. Habershon, D. E. Manolopoulos, T. E. Markland, and T. F. Miller III, Annu. Rev. Phys. Chem., 2013, 64, $387-413$.

[12] A. R. Menzeleev, F. Bell, and T. F. Miller III, J. Chem. Phys., 2014, 140, 064103-17.

[13] P. Ehrenfest, Z. Phys., 1927, 45, 455457.

[14] J. C. Tully, J. Chem. Phys., 1990, 93, 1061-1071.

[15] J. C. Tully, in Classical and Quantum Dynamics in Condensed Phase Simulations, ed. B. J. Berne, G. Ciccotti, and D. F. Coker, World Scientific, 1998; pp. 34-71.

[16] J. C. Tully, Mixed Quantum-Classical Dynamics, Modern Methods for Multidimensional Dynamics Computations in Chemistry, 1998.

[17] J. C. Tully, J. Chem. Phys., 2012, (137), 22A301-7.

[18] J. E. Subotnik, W. Ouyang, and B. R. Landry, J. Chem. Phys., 2013, 139, 214107-16.

[19] V. Parandekar and J. C. Tully, J. Chem. Phys., 2005, 122, 094102.

[20] J. R. Schmidt, N. Shenvi, and J. C. Tully, J. Chem. Phys., 2008, 129, 114110-6.

[21] B. R. Landry and J. E. Subotnik, J. Chem. Phys., 2011, 135, 191101-4.

[22] B. R. Landry and J. E. Subotnik, J. Chem. Phys., 2012, 137, 22A513-13.

[23] M. C. Sherman and S. A. Corcelli, J. Chem. Phys., 2015, 142, 024110-8.

[24] R. A. Marcus, J. Chem. Phys., 1956, 24, 966-978.

[25] R. A. Marcus, Rev. Mod. Phys., 1993, 65, 599-610.

[26] B. S. Brunschwig, J. Logan, M. D. Newton, and N. Sutin, J. Am. Chem. Soc., 1980, 102, 5798-5809.

[27] M. D. Newton and N. Sutin, Annu. Rev. Phys. Chem., 1984, 35, 437-480.

[28] A. Nitzan, Chemical Dynamics in Condensed Phases, Oxford University Press, 2006.

[29] H. Eyring, J. Chem. Phys., 1935, 3, 107-115.

[30] L. D. Landau, Phys. Z. Sowjetunion, 1932, 1, 88.

[31] C. Zener, Proc. R. Soc. London, Ser. A, 1932, 137, 696-702.

[32] J. Spencer, F. Gajdos, and J. Blumberger, J. Chem. Phys., 2016, under review.

[33] T. Kubař and M. Elstner, Phys. Chem. Chem. Phys., 2013, 15, 5794-5813. 
[34] T. Kubař and M. Elstner, J. R. Soc. Interface, 2013, 10, 20130415.

[35] F. Gajdos, S. Valner, F. Hoffmann, J. Spencer, M. Breuer, A. Kubas, M. Dupuis, and J. Blumberger, J. Chem. Theory Comput., 2014, 10, 4653.

[36] B. R. Landry, M. J. Falk, and J. E. Subotnik, J. Chem. Phys., 2013, 139, 211101-4.

[37] M. D. Newton, Chem. Rev., 1991, 91, 767-792.

[38] J. Blumberger, Chem. Rev., 2015, 115, 11191-11238.

[39] H. Oberhofer and J. Blumberger, Phys. Chem. Chem. Phys., 2012, 14, 13846-13852.

[40] F. Gajdos, H. Oberhofer, M. Dupuis, and J. Blumberger, J. Phys. Chem. Lett., 2013, 4, 1012-1017.

[41] K. P. McKenna, M. J. Wolf, A. L. Shluger, S. Lany, and A. Zunger, Phys. Rev. Lett., 2012, $108,116403$.

[42] J. Blumberger and K. McKenna, Phys. Chem. Chem. Phys., 2013, 15, 2184-2196.

[43] D. A. Case, T. Darden, T. Cheatham, III, C. Simmerling, J. Wang, R. Duke, R. Luo, M. Crowley, R. C. Walker, and W. Zhang, et al., AMBER 10, University of California, San Francisco, 2008.

[44] O. V. Prezhdo and P. J. Rossky, J. Chem. Phys., 1997, 107, 5863-5878.

[45] J. Y. Fang and S. Hammes-Schiffer, J. Phys. Chem. A, 1999, 103, 9399-9407.

[46] C. Zhu, S. Nangia, A. W. Jasper, and D. G. Truhlar, J. Chem. Phys., 2004, 121, 7658-7670.

[47] C. Zhu, A. W. Jasper, and D. G. Truhlar, J. Chem. Theory Comput., 2005, 1, 527-540.

[48] A. W. Jasper, S. Nangia, C. Zhu, and D. G. Truhlar, Acc. Chem. Res., 2006, 39, 101-108.

[49] G. Granucci and M. Persico, J. Chem. Phys., 2007, 126, 134114-11.

[50] N. Shenvi, J. E. Subotnik, and W. Yang, J. Chem. Phys., 2011, 134, 144102-12.

[51] A. E. Sifain, L. Wang, and O. V. Prezhdo, J. Chem. Phys., 2016, 144, 211102.

[52] J. J. Kwiatkowski, J. M. Frost, and J. Nelson, Nano Lett., 2009, 9, 1085.

[53] A. Troisi, D. L. Cheung, and D. Andrienko, Phys. Rev. Lett., 2009, 102, 116602.

[54] D. V. Matyushov, J. Chem. Phys., 2013, 139, 025102-12. 
Table 1: Parameterisation of reorganisation energy $\lambda$, Eq. 31, via the equilibrium bond length of the $\mathrm{C}=\mathrm{C}$ bond of the charged ethylene-like molecule; data used for Figure 3.

$\begin{array}{cr}\text { Bond length }(\AA) & \lambda(\mathrm{meV}) \\ 1.324 & 0 \\ 1.369 & 100 \\ 1.387 & 200 \\ 1.401 & 300 \\ 1.413 & 400 \\ 1.423 & 500 \\ 1.433 & 600 \\ 1.441 & 700\end{array}$


Table 2: Parameterisation of electronic coupling $H_{\mathrm{ab}}$ via the constant $C$, Eq. 5 .

$\begin{array}{lc}C(\mathrm{meV}) & \left\langle\left|H_{\mathrm{ab}}\right|^{2}\right\rangle^{1 / 2}(\mathrm{meV})^{a} \\ 89 & 8 \\ 133 & 11 \\ 177 & 15 \\ 266 & 23 \\ 355 & 30 \\ 532 & 45 \\ 710 & 61 \\ 887 & 76 \\ 1330 & 114 \\ 1774 & 151 \\ 2217 & 189 \\ 2661 & 227 \\ 3104 & 265 \\ 3548 & 303 \\ 3991 & 341 \\ 4435 & 378 \\ 4878 & 418 \\ 5322 & 455\end{array}$

${ }^{a}$ Averaged over an MD trajectory in the initial diabatic state. Data are used for Figure 5. 

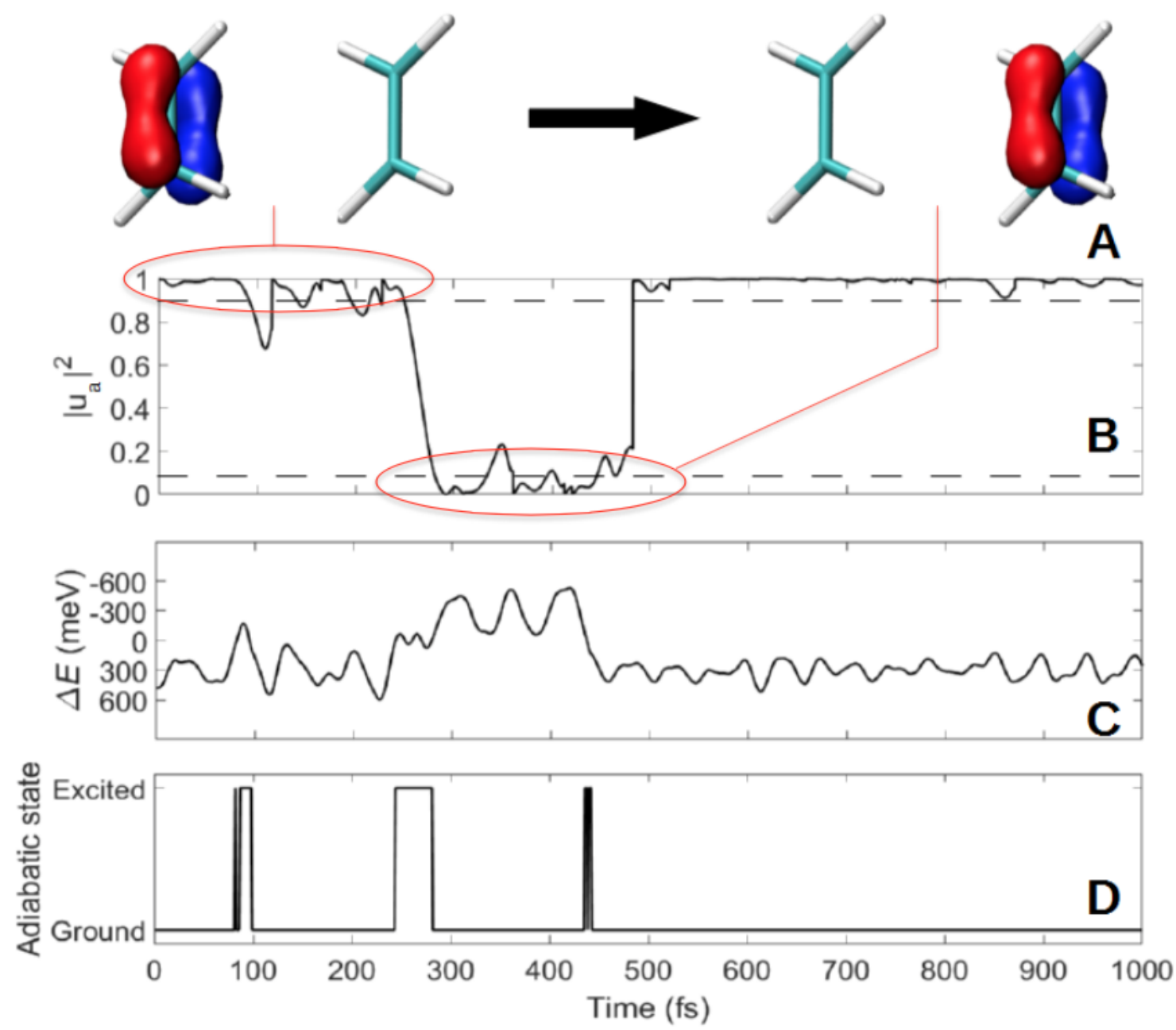

Figure 1: An illustration of the donor-acceptor system investigated comprising of two ethylene-like molecules (ELM) and the behaviour of a typical SH trajectory. Panel A shows a pictorial representation of an excess hole transferring from donor (left, initial state "a") to acceptor (right, final state "b"). Panels B, C and D are time series of different properties during a typical FOB-SH simulation of hole transfer. Panel B shows the time evolution of the initial state amplitude $\left|u_{\mathrm{a}}\right|^{2}$, including a transfer from initial to final state and back. Panel C shows the time evolution of the diabatic energy gap $\Delta E$, Eq. 10, and panel D shows the active adiabatic electronic state of the system. 


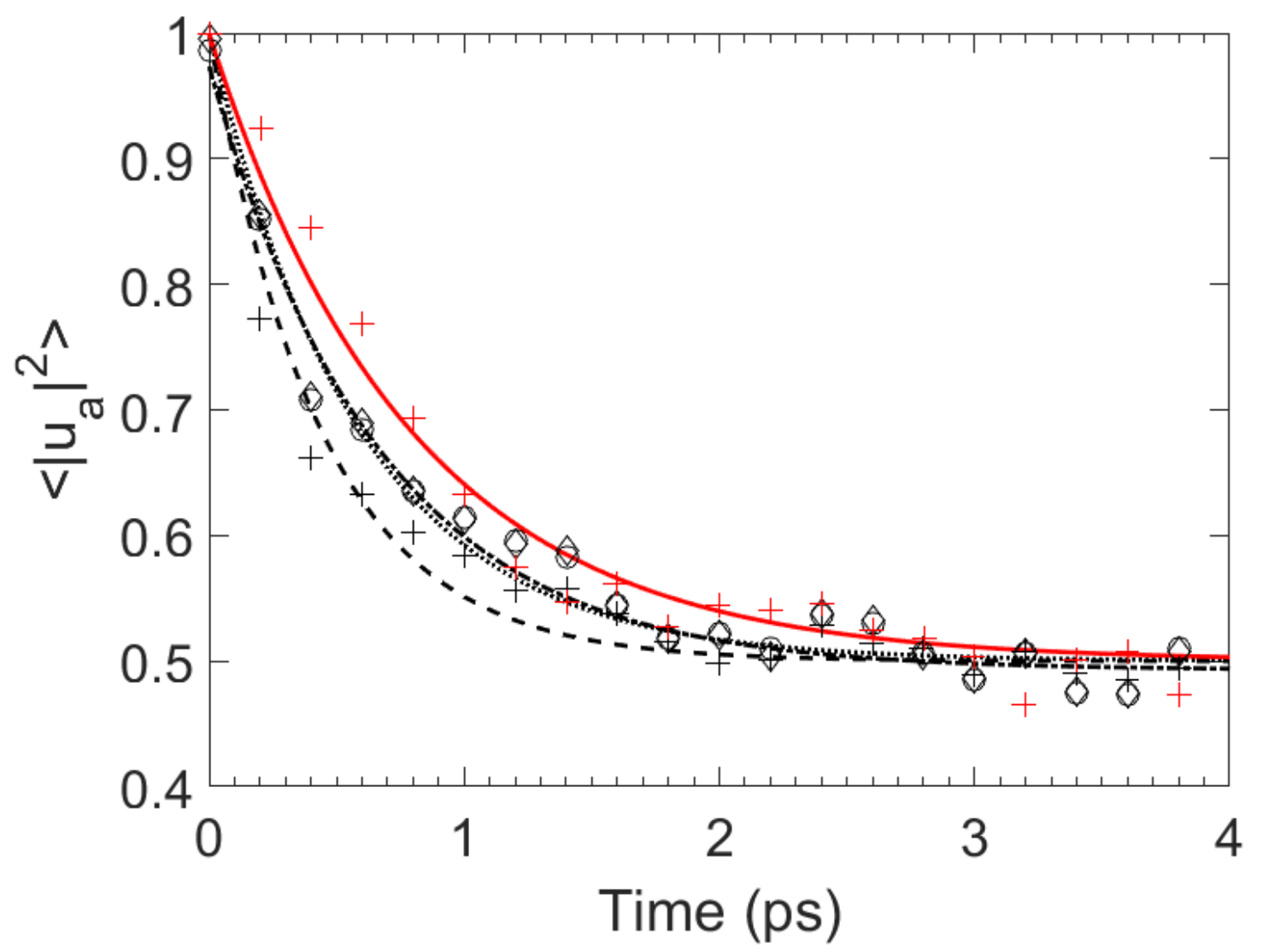

Figure 2: A comparison of the population decay calculated with the three methods in Ref. ${ }^{36}$ and fitted to an exponential Eq. 15: method 1 (circles, dotted line), method 2 (Eq. 14, plus symbols, dashed line) and method 3 (diamonds, dot dashed line). We have additionally plotted the population decay calculated via method 2 with the decoherence correction, with an exponential fit as a solid red line. Simulations were carried out for $\lambda=0.1 \mathrm{eV}$, $\left\langle\left|H_{\mathrm{ab}}\right|^{2}\right\rangle^{1 / 2}=8 \mathrm{meV}$ and $\Delta A=0$. 


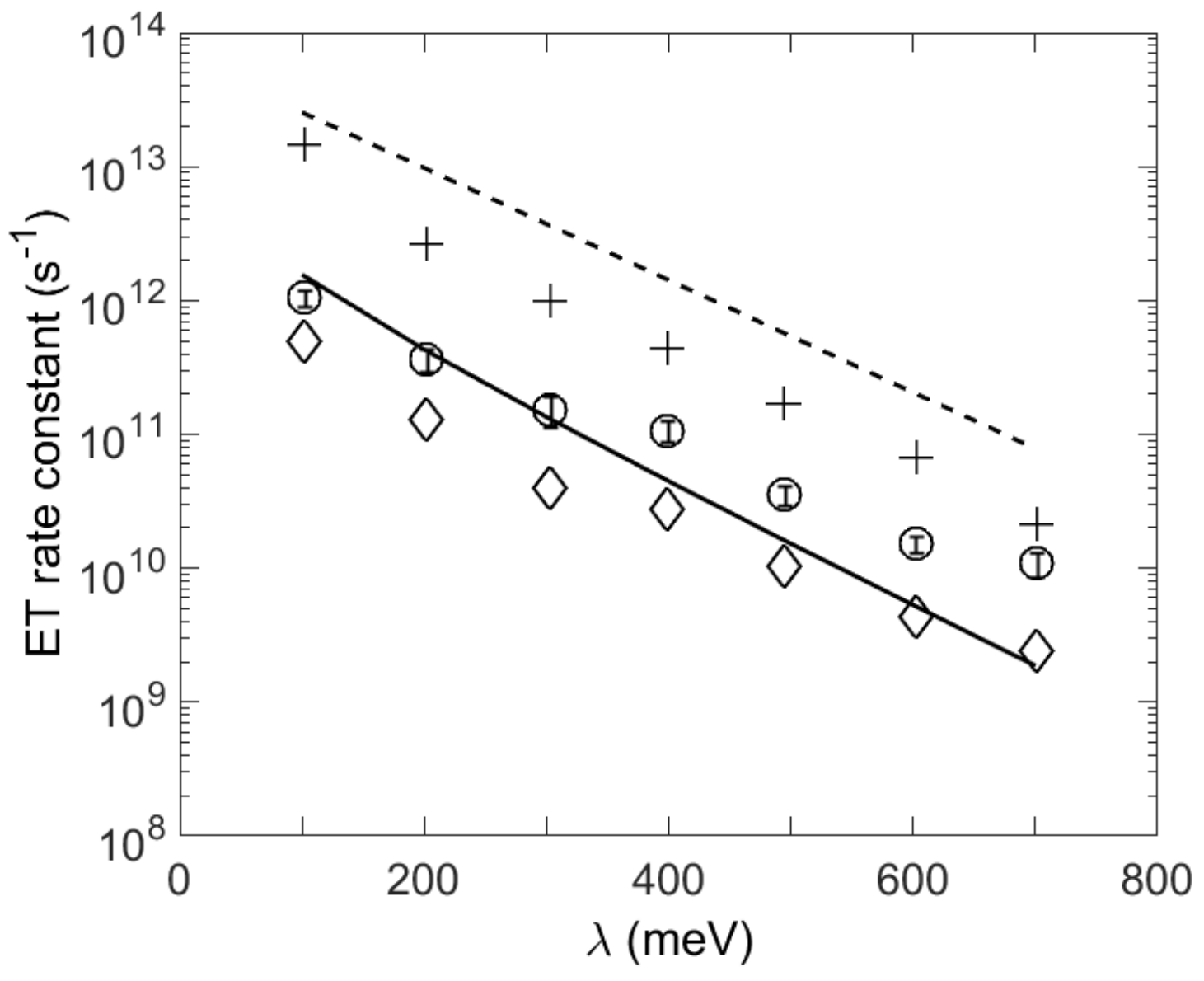

Figure 3: ET rates against reorganisation energy $\lambda$, obtained from FOB-SH simulations via population decay, Eq. 18 (circles), and transition count, Eq. 19 (diamonds). For comparison, the ET rate from semi-classical ET theory, Eq. 20, is drawn in solid lines. We additionally plot the rate of nuclear transitions from initial to final diabatic state minimum as measured by the energy gap Eq. 10 (plus symbols) and the adiabatic ET rate, Eq. 30 (dashed line), to understand the contribution of nuclear and electronic transitions to the ET rate. Simulations were carried out for $\left\langle\left|H_{\mathrm{ab}}\right|^{2}\right\rangle^{1 / 2}=8 \mathrm{meV}$ and $\Delta A=0$. 

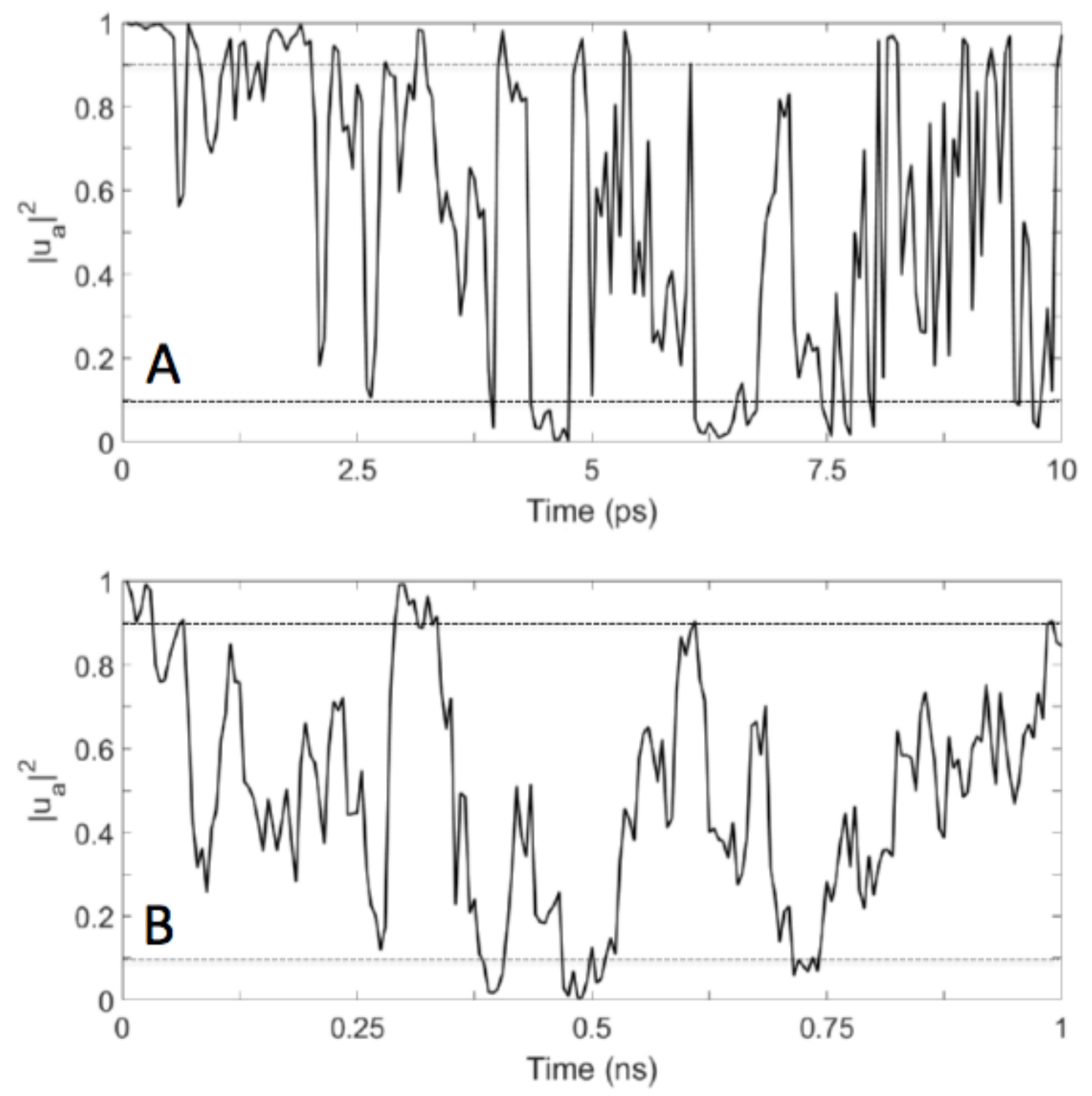

Figure 4: A comparison of the different electronic behaviour for two different values of reorganisation energy $\lambda$. In panel $\mathrm{A}, \lambda=0.1 \mathrm{eV}$ and we observe many large-amplitude oscillations of the initial electronic state amplitude $\left|u_{\mathrm{a}}\right|^{2}$ within $10 \mathrm{ps}$, including a number of successful transitions as indicated by dashed lines. In contrast, panel B has $\lambda=0.7 \mathrm{eV}$ and over the course of $1 \mathrm{~ns}$ there are only few successful transitions, while for long periods of time the population becomes stuck in a half-and-half state, contributing to the rate by exponential decay but not the rate by transition count. Resetting of the electronic populations as part of the decoherence correction may not be visible due to the finite stride of points plotted. Data are plotted for a SH trajectory that was used to obtain the ET rates shown in Fig. 3. 

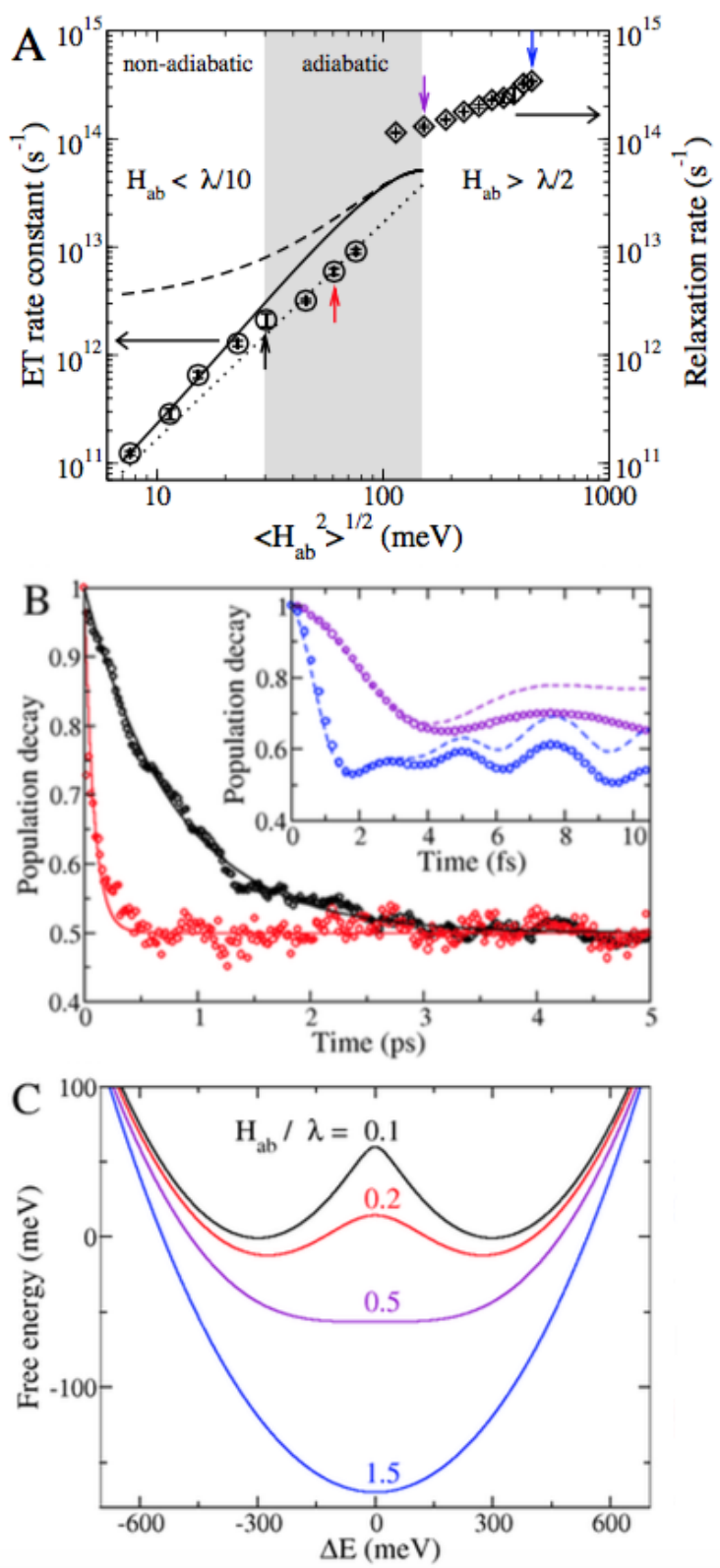

Figure 5: (A) ET rates from FOB-SH MD simulation ( $k_{\mathrm{p}}^{\mathrm{SH}}$, Eq. 18) against average diabatic electronic coupling $\left\langle\left|H_{\mathrm{ab}}\right|^{2}\right\rangle^{1 / 2}$ (circles). For comparison, the ET rates from semi-classical ET theory, Eq. 20 (solid lines), and the respective non-adiabatic limit Eq. 29 (dotted line) and adiabatic limit Eq. 30 (dashed lines) are displayed. For large electronic coupling, charge relaxation rates from FOB-SH MD simulation are shown $\left(t_{\max }^{-1}\right.$, diamonds). (B) Exponential fits (Eq. 15, solid line) to the initial diabatic state population decay (Eq. 14, circles) are shown for electronic coupling values marked by black and red vertical arrows in panel (A). The inset displays the population decay (circles) at large electronic coupling values marked by purple and blue vertical arrows in panel (A). The data in the inset are fit to thermally averaged Rabi oscillations (Eq. 32, dashed lines). (C) Free energy profiles of the adiabatic electronic ground state, Eq. 24, for the four coupling values marked by arrows in panel (A). All FOB-SH MD simulations were carried out for $\lambda=0.3 \mathrm{eV}$ and $\Delta A=0$. 


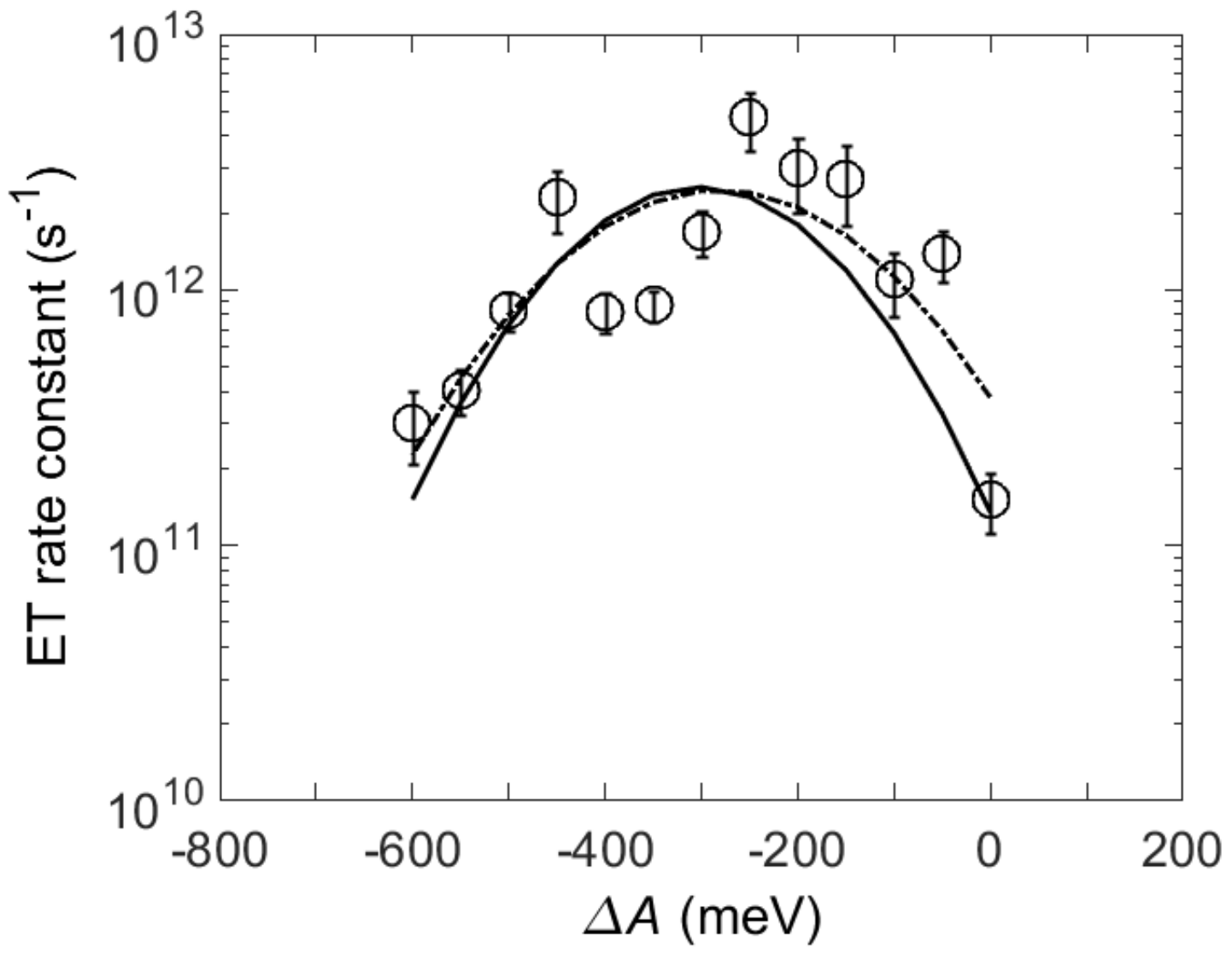

Figure 6: ET rates against driving force (ET free energy difference), $\Delta A$, obtained from FOB-SH simulations via population decay, Eq. 18 (circles). A best fit to an inverse parabola is shown in dash-dotted lines. For comparison, ET rates from the semi-classical ET theory, Eq. 20, are shown in solid lines. Simulations were carried out for $\lambda=0.3 \mathrm{eV}$ and $\left\langle\left|H_{\mathrm{ab}}\right|^{2}\right\rangle^{1 / 2}=8 \mathrm{meV}$. 